\title{
On Coalition Incentive Compatibility
}

\author{
JERRY GREEN \\ Harvard University \\ and \\ JEAN-JACQUES LAFFONT \\ Ecole Polytechnique
}

\section{INTRODUCTION}

Progress has recently been made in the study of mechanisms designed to elicit, from economic agents, truthful revelation of their characteristics. The relevance of these results is best illustrated with reference to the free rider problem which occurs in public decision making. The Government must know agents' preferences to choose the best public projects, but it may be in agents' interest to provide distorted reports on their preferences.

A class of mechanisms has been proposed by Vickrey (1961), Clarke (1971), Groves (1973) which has the property that, in environments with separable utility functions, truthful revelation of preferences is a dominant strategy for each individual and a Pareto optimal decision is taken. In this context, since unrestricted transfers of a private resource are allowed, Pareto optimality is interpreted to mean that the public decision taken is the same as that which would be associated with all Pareto optimal resource allocations. Alternatively, recognizing that the mechanism may engender a surplus or a deficit, it is natural to introduce an artificial agent (the government or planner) capable of absorbing this imbalance. Using the net transfer to this agent as his utility function, Pareto optimality can be interpreted in the usual manner for the enlarged set of agents. The impossibility of closing the mechanism while maintaining optimality and incentive compatibility has been proven by Hurwicz (1975), Green and Laffont (1976a) and Walker (1976) in various contexts. In Green and Laffont (1975) we have shown that the class of mechanisms proposed by Groves includes all such mechanisms. Furthermore, allowing more general mechanisms, we have proved that any satisfactory mechanism (i.e. any mechanism which takes Pareto optimal decisions and for which dominant strategies exist) is isomorphic to a Groves mechanism.

The incentive compatibility obtained with these mechanisms applies to individuals; for each agent the truth is his best possible answer for any willingnesses to pay professed by the others. Even though it was soon realized (see Bennett and Conn (1976), Groves (1973) and Vickrey (1961)) that these mechanisms are vulnerable to manipulation by coalitions no analytical attempt to ameliorate this situation has yet been made. In Green and Laffont (1976b) we suggested the use of a sampling version of Groves mechanisms to mitigate this coalition problem by making cooperation very costly and unlikely. The purpose of this paper is to look more carefully into this question.

If the structure of binding coalitions forms a partition of the agents and is known to the decision maker, then, by treating each coalition as a single agent, it is still possible to induce truthful revelation. When the decision maker does not know this structure, or when, a priori, any structure is possible, the mechanism must be designed to be incentive compatible for any coalition. This is clearly impossible. Should we then say that no positive result concerning coalition incentive compatibility can be obtained for Groves mechanisms? 
The main purpose of this paper is to show that there is a sense in which Groves mechanisms are somewhat robust to coalition manipulations.

In Section 2, we set up the model and provide definitions. Section 3 is devoted to a formal proof of the impossibility of finding coalition incentive compatible Groves mechanisms, even if only a single coalition of two or more agents can form. Then we consider a particular Groves mechanism, the pivotal mechanism, which has been found especially interesting in other contexts (Green and Laffont $(1976 a, b)$. Section 4 explains how a coalition of size $n$ will distort its reported preferences when faced with the pivotal mechanism. In Section 5, we show that for a fixed coalition the expected gain to cheating compared to telling the truth decreases with the number of agents in the population. Then, considering the population as a random sample in a fixed distribution, we obtain that the probability that a cheating coalition (of size less than the square root of the population size) will exist with a per-capita gain larger than any fixed positive number approaches zero as the sample size grows. Generalizations to other mechanisms are given in Section 6. Finally, in Section 7 we provide an informal discussion of these results which points out the sense in which coalition manipulation can therefore be somewhat mitigated.

\section{DEFINITIONS}

For simplicity, we consider a fixed size project of zero cost. Costly projects can be handled by considering evaluations net of imputed cost shares. The utility function of agent $i$ (for $i=1, \ldots, N$ ) is:

$$
\begin{aligned}
u_{i} & =x_{i}+v_{i} & & \text { if the project is realized } \\
& =x_{i} & & \text { if the project is not realized }
\end{aligned}
$$

where $x_{i}$ is the amount of a transferable private good consumed by individual $i$ and $v_{i}$ is the true willingness to pay of the agent for the public project.

Let $w_{i}$ be agent $i$ 's stated willingness to pay, for $i=1, \ldots, N$. Let $w=\left(w_{1}, \ldots, w_{N}\right)$.

Definition 1. A revelation mechanism is a mapping $f$

$$
f: R^{N} \rightarrow\{0,1\} \times R^{N} \quad \text { written as } f\left(w_{1}, \ldots, w_{N}\right)=\left(d, t_{1}, \ldots, t_{N}\right)
$$

where $d \in\{0,1\}$ is the public decision taken $(1=$ accept project; $0=$ reject project $)$ and $t_{i}$ is the transfer of the private good received by agent $i$.

The answer $w_{i}$ given by individual $i$ is obtained from a maximization programme; for example the maximization of his expected utility for given expectations about the stated willingnesses to pay of others.

Definition 2. A revelation mechanism is successful if

$$
d(w)=1 \leftrightarrow \sum_{i} v_{i} \geqq 0 .
$$

Definition 3. A revelation mechanism is strongly individually incentive compatible, s.i.i.c., if $v_{i}$ is a dominant strategy for each agent.

Definition 4. A Groves mechanism is a revelation mechanism such that:

$$
\begin{aligned}
& t_{i}(w)=\sum_{j \neq i} w_{j}+h_{i}\left(w \mid w_{i}\right) \text { if } \sum_{i} w_{i} \geqq 0 \\
& =h_{i}\left(w \backslash w_{i}\right) \quad \text { if } \sum_{i} w_{i}<0
\end{aligned}
$$

where $h_{i}($.$) is an arbitrary function, i=1, \ldots, N$ and the notation $\left(w \mid w_{i}\right)$ means $\left(w_{1}, \ldots, w_{i-1}, w_{i+1}, \ldots, w_{N}\right)$.

In Green and Laffont (1975) we have shown that a s.i.i.c. and successful revelation mechanism has to be a Groves mechanism. Is it possible to restrict further the functions 
$h_{i}($.$) to avoid, even partly, manipulation by coalitions? The next section gives a negative$ answer.

\section{AN IMPOSSIBILITY THEOREM}

Let $C$ be a coalition of agents. The vector $v_{C}$ will denote the appropriate projection of $v \in R^{N}$, the true willingnesses to pay. Let $w \in R^{N}$ be a vector of professed willingnesses to pay. We use $\left(w \backslash w_{C}\right)$ to denote the components of $w$ with the exception of $w_{i}, i \in C$ and the notation $w=\left(w_{N \backslash C}, w_{C}\right)$.

The payoff function of a coalition $C$ is

$$
\begin{aligned}
& U_{C}(w)=\sum_{i \in C} t_{i}(w)+\sum_{i \in C} v_{i} \text { if } d=1 \\
& =\sum_{i \in C} t_{i}(w) \quad \text { if } d=0 .
\end{aligned}
$$

if

Definition 5: A revelation mechanism is strongly coalition incentive compatible (s.c.i.c.)

$\forall C, v_{C}$ is a dominant strategy for the coalition $C$, i.e.

$$
U_{C}\left(w_{N \mid C}, v_{C}\right) \geqq U_{C}\left(w_{M \backslash C}, w_{C}\right), \forall w_{N \mid C}, \forall w_{C} .
$$

Theorem 3.1. There exists no s.c.i.c. successful revelation mechanism. (Proved independently by Bennett and Conn (1976)).

Therefore, if we allow any type of coalitions and in particular size 2 coalitions, there exists no s.c.i.c. successful mechanism. However, if the partition of agents in coalitions is known in advance, it is easy to construct such a mechanism by treating each coalition as an agent. Is it possible to obtain a positive result by allowing for a restricted class of coalitions? This is an interesting question because, as we shall see in Section 4, the larger a deviant coalition is, the more it stands to gain from cooperative behaviour. Therefore, small coalitions are not the most severe problem. In any particular situation it might be that some coalitions size greater than two can be identified as the one which presents the greatest danger in terms of cooperative strategic behaviour. If mechanisms existed that could avoid this problem, even though they failed to counter the possible cheating by two player coalitions, they still might be very valuable. The following theorem gives a negative answer to this question: no strongly individually incentive compatible mechanism exists that can avoid cheating by any other group of any size.

Let us assume that we require only the mechanism to be s.i.i.c. and immune to misrepresentation by a coalition of a fixed size say $n$ but not necessarily so with respect to others. In such a case we will say that the mechanism is size $n$-s.c.i.c.

The Bennett-Conn theorem above can be used to prove the non-existence of s.c.i.c. successful revelation mechanisms when two adjacent coalitions (i.e. of size $k$ and $k+1$ ) are allowed, which, of course, is in particular possible when all coalitions are allowable. If $n=2$, this result implies that there exists no size 2 s.c.i.c. successful revelation mechanism. For $n>2$, the proof is more delicate.

Theorem 3.2. For any $n>1$, there exists no successful revelation mechanism that is both s.i.i.c. and size $n$-s.c.i.c.

Proof. (From Green and Laffont (1977)), a s.i.i.c. successful revelation mechanism must be a Groves mechanism, i.e.

$$
\begin{aligned}
& t_{i}(w)=\sum_{j \neq i} w_{j}+h_{i}\left(w \backslash w_{i}\right) \quad \text { if } \quad \sum_{i} w_{i} \geqq 0, \quad i=1, \ldots, N \\
& =h_{i}\left(w \mid w_{i}\right) \quad \text { if } \sum_{i} w_{i}<0, \quad i=1, \ldots, N .
\end{aligned}
$$

Consider now a coalition $C$ of size $n$ and let us treat this coalition as a single agent called agent $C$. We have then an economy with $N-n+1$ agents. In this economy we 
can again apply the characterization theorem (Green and Laffont (1977)); we obtain the transfer function of " the agent $C$ ".

$$
\begin{aligned}
& t_{C}(w)=\sum_{j \notin C} w_{j}+h_{C}\left(w \backslash w_{C}\right) \text { if } \quad \sum_{i} w_{i} \geqq 0 \\
& =h_{c}\left(w \mid w_{c}\right) \quad \text { if } \sum_{i} w_{i}<0 .
\end{aligned}
$$

On the other hand $t_{C}(w)=\sum_{i \in C} t_{i}(w)$; from (3.1)

$$
\begin{aligned}
& t_{C}(w)=(n-1) \sum_{j \in C} w_{j}+n \sum_{j \notin C} w_{j}+\sum_{i \in C} h_{i}\left(w \mid w_{i}\right) \text { if } \quad \sum_{i} w_{i} \geqq 0 \\
& =\sum_{i \in c} h_{i}\left(w \mid w_{i}\right) \quad \text { if } \sum_{i} w_{i}<0 .
\end{aligned}
$$

From (3.2) (3.3) we have:

$$
\begin{array}{cc}
(n-1) \sum_{i} w_{i}+\sum_{i \in C} h_{i}\left(w \backslash w_{i}\right)=h_{C}\left(w \backslash w_{C}\right) & \text { if } \quad \sum_{i} w_{i} \geqq 0 . \\
\sum_{i \in C} h_{i}\left(w \mid w_{i}\right)=h_{C}\left(w \backslash w_{C}\right) & \text { if } \quad \sum_{i} w_{i}<0 .
\end{array}
$$

We are going to fix $w_{i}, i \notin C$, at $w_{i}^{0}$ so that $h_{C}\left(w \backslash w_{C}\right)$ will be a constant $K$.

For convenience we choose $w_{i}^{0}=0, i \notin C$. Consider now $w_{i}^{0}, i \in C$ and $A>0$, so that

$$
-(n-2) A>\sum_{i \in C} w_{l}^{0}>-(n-1) A .
$$

Since $w_{i}, i \notin C$, is fixed at $w_{i}^{0}$ in the whole proof we will delete in the $h$ functions the corresponding arguments, and we choose the indices of $w_{i}, i \in C$ as $i=1, \ldots, n$.

From (3.6) we have

$$
\begin{aligned}
\text { for } w_{i} & =w_{i}^{0}+A \quad i \in C, \quad i \neq n \\
w_{n} & =w_{n}^{0} \\
w & =\left(w_{1}^{0}+A, \ldots, w_{n-1}^{0}+A, w_{n}^{0}\right) \\
\sum_{i \in C} h_{i}\left(w \backslash w_{i}\right) & =-(n-1)\left[\sum_{i \in C} w_{i}^{0}+(n-1) A\right]+K .
\end{aligned}
$$

We consider now the $C_{n-1}^{1}$ combinations of $\left(w_{i}\right)$ such that

where

$$
\begin{aligned}
w_{n} & =w_{n}^{0} \\
w_{j} & =w_{j}^{0} \\
w_{i} & =w_{i}^{0}+A \quad i \neq j, \quad i \neq n,
\end{aligned}
$$

$$
C_{M}^{n}=\left(\begin{array}{c}
M \\
n
\end{array}\right)=\frac{M !}{n !(M-n) !}
$$

For each of them we have from (3.6):

$$
\sum_{i \in C} h_{i}\left(w \backslash w_{i}\right)=K
$$

The idea of the proof is to use these $C_{n-1}^{1}$ equations to suppress in (3.7) the terms which contain $(n-2)$ times $A$, with the exception of $h_{n}\left(w \backslash w_{n}\right)$.

This can be done by substracting the $(n-1)$ equations (3.8). By doing so we introduce $C_{n-1}^{1} \times(n-2)$ terms which contain $(n-3)$ times $A$.

We can then consider the $C_{n-1}^{2}$ combinations of $\left(w_{i}\right)$ such that

$$
\begin{aligned}
w_{n} & =w_{n}^{0} \\
w_{j} & =w_{j}^{0} \\
w_{j^{\prime}} & =w_{j^{\prime}}^{0} \\
w_{i} & =w_{i}^{0}+A \quad i \neq j, \quad i \neq n, \quad i \neq j^{\prime} .
\end{aligned}
$$


For each of them we have from (3.6):

$$
\sum_{i \in C} h_{i}\left(w \mid w_{i}\right)=K .
$$

In each equation (3.9) there are 2 terms with $(n-2) A$, i.e. for all the equations $C_{n-2}^{2} \times 2$ which is exactly $C_{n-1}^{1}(n-2)$, what we need (the fact that the $A$ are at the appropriate locations is obtained by symmetry) etc. . . .

At step $k$, we have $C_{n-1}^{k}(n-1-k)$ terms to eliminate, but we can write: $C_{n-1}^{k+1}$ equations, containing $C_{n-1}^{k+1}(k+1)$ terms, which are exactly the $C_{n-1}^{k}(n-1-k)$ terms that we need.

At step $(n-1)$, we introduce the terms with no $A$, which number

$$
C_{n-1}^{n-2} \times(n-2)=(n-1) \times(n-2) \text {. }
$$

By symmetry, we then have only to premultiply the equation

$$
\sum_{i \neq n, i \in C} h_{i}\left(w^{0} \mid w_{i}^{0}\right)+h_{n}\left(w^{0} \mid w_{n}^{0}\right)=K
$$

by $(n-2)$ to be able to subtract all the terms $h_{i}(),. i \neq n$ with no $A$.

Finally, we obtain an equation whose left hand side contains only terms in $h_{n}($.$) .$ This is an expression which does not depend on $w_{n}^{0}$ and whose right hand side is:

$$
-(n-1)\left[\sum_{i} w_{i}^{0}+(n-1) A\right]
$$

plus a constant, that is to say an expression which depends on $w_{n}^{0}$. Allowing $w_{n}^{0}$ to change in a neighbourhood without disturbing the inequalities (3.6) we therefore obtain a contradiction. \|

\section{OPTIMAL CHEATING BY A COALITION}

In this section and the next one, we are using a special Groves mechanism referred to as the pivotal mechanism (or Clarke mechanism) to show what coalitions can achieve by cheating. In Section 6 results are generalized to other Groves mechanisms.

The pivotal mechanism is obtained by specifying the $h($.$) function as follows:$

$$
\begin{aligned}
& h_{i}(w)=-\sum_{j \neq i} w_{j} \text { if } \sum_{j \neq i} w_{i} \geqq 0 \\
& =0 \quad \text { if } \quad \sum_{j \neq i} w_{i}<0 .
\end{aligned}
$$

With such a mechanism, agents are never subsidized and may be taxed but still have an incentive to participate (see Green and Laffont (1976b)).

It can easily be seen that if a coalition of individuals forms, and if, tacitly or explicitly, these individuals agree to alter their responses in the same direction, a superior result from their point of view can sometimes be achieved. A simple example of this can be obtained in a three person economy with $v_{1}=-3, v_{2}=+1, v_{3}=+1$. Using any Groves mechanism each agent has an incentive to respond with $w_{i}=v_{i}$. The project would be rejected but if individuals 2 and 3 could agree to each say +3 , then the pivotal mechanism would lead to acceptance with no transfers, and this is better than they would achieve by telling the truth. However they cannot be assumed to know the statement that will be made by the other individuals; or, more generally, they will even be ignorant of how many other individuals there are. Distortions will involve some risks. For example, if $w_{1}=-5$, then the distortion mentioned above would lead to both individuals being taxed 2 units by the pivotal mechanism, which is more than their true willingness to pay for the project they have caused to be accepted. The larger their distortion, the surer they are of forcing their preferred social decision, but the more risk they must accept if their guess about the others' statements proves erroneous.

To formalize this trade-off, let us consider a coalition $C$ of size $n$. There are $N-n$ other individuals. We assume that everybody in the coalition has the same expectations on the sum of the answers by the $(N-n)$ others; these expectations are represented by a 
strictly increasing probability distribution function $F_{N}(x)$. The associated density function is $f_{N}($.$) .$

Let $w_{i}$ be the answer given by the agent $i$ for $i$ in $C$. Let $V=\sum_{i \in C} v_{i}$ and $W=\sum_{i \in C} w_{i}$. We will call these the global true value and the global answer for coalition $C$. This formulation of the problem presumes that the coalition in question can enforce its optimal strategy to be played by each of the colluding members. Of course, coalitions of this type may very well have free rider problems of their own. For this reason it is natural to be concerned primarily with smaller coalitions, and indeed the main theorem of this paper (see Section 5) is that small profitably colluding coalitions will not be likely to form in a large economy. By neglecting the incentive problems within deviant coalitions, we are taking the most pessimistic stance and hence the result is strengthened. To the extent that coalitions cannot enforce their cooperatively optimal behaviour, individual incentives, which favour truthtelling, will dominate.

For the coalition $C$, the expected utility of saying $w_{C}=\left(w_{i}\right)_{i \in C}$ is:

$$
U\left(N, n, w_{C}\right)=V \int_{-W}^{\infty} d F_{N}(x)+\sum_{i \in C} \int_{-\Sigma_{j \in C} w_{j}}^{-\Sigma_{j e c} j \neq i w_{j}}\left[x+\sum_{j \in C, j \neq i} w_{j}\right] d F_{N}(x)
$$

which is decomposed in the expected utility of getting the project minus the expected tax.

The answer of a coalition is symmetric if everybody in the coalition gives the same personal evaluation.

Theorem 4.1. For any global answer $W$ by the coalition, the optimal answer of the coalition is symmetric.

Proof. Let us consider the programme

$$
\begin{aligned}
& \max _{\left(w_{C}\right)} U\left(N, n, w_{C}\right) \\
& \sum_{i \in C} w_{i}=W .
\end{aligned}
$$

(4.1) can be rewritten as

$$
\max _{\left(w_{c}\right)} V \int_{-W}^{\infty} d F_{N}(x)+\sum_{i \in C} \int_{-W}^{-W+w_{i}}\left[x+W-w_{i}\right] d F_{N}(x)
$$

subject to $\sum_{i \in C} w_{i}=W$.

The first order conditions are ( $\lambda$ being the Lagrange multiplier)

$$
-\int_{-W}^{-W+w_{i}} d F_{N}(x)=\lambda \quad i \in C .
$$

For any $\left(j, j^{\prime}\right) \in C,(4.3)$ gives

or

$$
F_{N}\left(W+w_{j}\right)=F_{N}\left(W+w_{j}\right)
$$

$$
w_{j}=w_{j} \cdot
$$

It is easy to see that the second order conditions are fulfilled. \|

Let $w$ be the common individual answer of the members of the coalition $C . W=n w$. The expected utility gain of saying $W$ instead of the truth $V$ is:

$$
\begin{aligned}
G(N, n)=V \int_{-W}^{-V} d F_{N}(x)+n \int_{-W}^{-W(n-1) / n}[ & x+((n-1) n) W] d F_{N}(x) \\
& -\sum_{i \in C} \int_{-V}^{-V+v_{i}}\left[x+\sum_{j \neq i} v_{j}\right] d F_{N}(x) .
\end{aligned}
$$

The first term represents the utility gained from the fact that the project will be accepted in some circumstances in which it would not otherwise be, or vice versa, because of this 
coalition's exaggeration of its beliefs. The second term is the expected pivotal payment that the coalition will make. Since they answer symmetrically they will all be taxed in a like manner, when $x \in[-n w,-(n-1) w]$. Finally, the last term is the pivotal payments they would have had to pay had they answered truthfully; these pivotal payments are now avoided.

Theorem 4.2. If $\sqrt{N} f_{N}(-n w)$ converges to a constant as $N$ goes to infinity, and $\sqrt{N} f_{N}^{\prime}(y)$ is continuous in $y$ and for each y converges to zero, then $w$ converges to $V$ as $N$ goes to infinity (and therefore $W$ goes to $n V$ ).

Proof. The optimal answer for each $N$ is obtained by maximizing $G(N, n)$, i.e. from

$$
V f_{N}(-W)+(n-1) \int_{-W}^{-W+(W / n)} d F_{N}(x)-W f_{N}(-W)=0
$$

which can be rewritten as:

$$
(V-n w) f_{N}(-n w)+(n-1) w f_{N}(-n w+\mu w)=0 \text { for some } \mu \in[0,1]
$$

or, by the Mean Value Theorem, as:

$$
(V-w) f_{N}(-n w)+(n-1) \mu w^{2} f_{N}^{\prime}(-n w+\tilde{\mu} w)=0 \text { for some } \tilde{\mu} \in[0, \mu] .
$$

Multiplying (4.7) by $\sqrt{N}$ we obtain the result directly from the assumptions. \|

The somewhat technical assumptions of Theorem 4.2 are indeed quite general and in particular are satisfied by the normal distribution with mean zero (used below). In the next section, we will assume that $N$ is large enough to validate the approximation of $W$ by $n V$, i.e. that the optimal way of cheating is for each member of a coalition of size $n$ to say $n$ times the average true willingness to pay in the coalition.

It is interesting to observe that the optimal cheating answers depend on the size of the coalition and that the answer should be the same for every member of the coalition.

Because of the second point they will all be or not be pivotal together, which at least for identical agents makes side-payments non-necessary. The first point makes implicit coalition formation without communication much more difficult, because they have to guess the size of the group with which they are tacitly colluding.

\section{VALUE OF OPTIMAL CHEATING}

In this section we restrict ourselves to the case of a distribution function $F_{N}($.$) , which is$ normal with mean zero and variance $N$. A more general theory would be much more technical without throwing additional light on the basic result.

The expected gain (4.4) of cheating for a coalition of size $n$ can then be bounded as follows (see Appendix).

$$
\begin{aligned}
G_{C}(V, N) \leqq \frac{(n-1) V^{2}}{\sqrt{2 \pi N}} & \cdot e^{-\frac{1}{2} \frac{V^{2}}{N}}+\frac{n(n-1)}{\sqrt{2 \pi N}} V^{2} e^{-\frac{1}{2} \frac{(n-1)^{2} V^{2}}{N}} \\
+ & \frac{n \sqrt{N}}{\sqrt{2 \pi}}\left[e^{-\frac{n^{2} V^{2}}{2 N}}-e^{-\frac{(n-1)^{2}}{2 N} \cdot V^{2}}\right] \\
& -\frac{(n-1) V^{2}}{n \sqrt{2 \pi N}} \cdot e^{-\frac{V^{2}}{2 N}}-\frac{n \sqrt{N}}{\sqrt{2 \pi}}\left[e^{-\frac{V^{2}}{2 N}}-e^{-\left(\frac{n-1}{n}\right)^{2} \frac{V^{2}}{2 N}}\right] .
\end{aligned}
$$

From this expression it is clear that for a fixed coalition (that is to say a fixed $n$ and a fixed $V$ ), the expected gain of cheating can be made as small as we wish, for $N$ large enough. 
However, when we increase the size of the population, the probability that, keeping $n$ constant, there exists a coalition of size $n$ with a very large $V$ increases if we view the members of the population as being drawn independently from the same distribution as they themselves believe. Therefore, we would like to show a stronger result, namely, that, for any $n$, the probability that there will exist a coalition of size $n$ such that its gain from cheating is larger than a given number, can be made as small as we wish by increasing $N$. Indeed, we have a stronger result:

Theorem 5.1. Let $\varepsilon$ and $\delta$ be positive real numbers, and let $n$ be a positive integer. There exists a population size, $N_{0}$, sufficiently large such that for all populations of size $N>N_{0}$, the probability that there exists a coalition of size $n<N^{\frac{1}{\delta}-\delta}$ within this population, capable of giving each of its members an increment of at least $\varepsilon$ in expected utility by cheating optimally, can be made arbitrarily small.

Proof. Note first that the global willingness to pay, $V$, of a potential cheating coalition is such that $V \leqq n v_{\max }$, where $v_{\max }$ is the maximum willingness to pay in the entire population.

Considering the population as a random sample of size $N$ in a normal distribution $\mathscr{N}(0,1)$, we argue now that for every fixed probability $\eta>0$, for every $\delta>0$,

$$
\text { prob }\left[v_{\max }>N^{\delta}\right]<\eta \text { for } N \text { large enough. }
$$

From David (1970), we know that the asymptotic distribution of the random variable $M_{n}=(2 \log N)^{\frac{1}{2}}\left[v_{\max }-(2 \log N)^{\frac{1}{2}}\right]$ is such that

$$
\operatorname{prob}\left[M_{n} \leqq v\right]=e^{-e^{-v}} \text {. }
$$

Therefore for $N$ large enough we have that

$$
\text { prob }\left[v_{\max }<v(2 \log N)^{-\frac{1}{2}}+(2 \log N)^{\frac{1}{t}}\right] \approx e^{-e^{-v}} .
$$

Letting $v=(2 \log N)^{\frac{1}{4}}\left[N^{\delta}-(2 \log N)^{\frac{1}{2}}\right]$, we obtain

$$
\text { prob }\left[v_{\max }<N^{\delta}\right] \approx e^{-e^{-\left[(2 \log N)^{\ddagger}\left(N^{\delta}-2 ! \log N\right)^{\ddagger}\right]}} \text {. }
$$

Clearly, (5.2) is then valid for large $N$.

Taking $n \leqq N^{\frac{1}{6}-\delta}$, we have $V<N^{\frac{t}{6}-\delta}$. $v_{\max }$, which implies with (5.2) that with probability $1-\eta$

$$
V<N^{t} \text {. }
$$

From (5.1) and the appendix, we know that the per-capita gain is of the order of $n V^{2} / N^{\frac{1}{2}}$. From (5.4) the per-capita gain is less than $N^{-\delta}$ which converges to zero as $N$ grows. Thus as long as $V<N^{\delta}$, which we know will happen with probability $1-\eta$, the conclusion of the theorems holds. \|

\section{GENERALIZATIONS}

In this section we extend the results to a subclass of Groves mechanisms.

Let $c(w)$ be the $h(\cdot)$ function corresponding to the pivotal mechanism

$$
\begin{aligned}
& c(w)=-\sum_{j \neq i} w_{j} \text { if } \quad \sum_{j \neq i} w_{j} \geqq 0 \\
& =0 \quad \sum_{j \neq i} w_{j}<0 .
\end{aligned}
$$

We write the $h(\cdot)$ function of any Groves mechanism as the sum of $c(w)$ and a function $\delta(w)$ which therefore characterizes also the given Groves mechanism

$$
\forall j, h_{j}(w)=c(w)+\delta_{j}\left(w \mid w_{j}\right) . \quad \forall w_{-j} .
$$

A Groves mechanism is said to be impersonal, if for every $j, \delta_{j}\left(w \mid w_{j}\right)$ is the same symmetric function of its argument. 
A Groves mechanism is said to be concave differentiable if for every $j, \delta_{j}\left(w \backslash w_{j}\right)$ is a concave differentiable function of $w_{-j}$.

Theorem 6.1. For any concave differentiable impersonal Groves mechanism and for any desired aggregate answer $W$ by a coalition, the optimal answer of a coalition is symmetric.

Proof. The objective function of the coalition is now

$$
\begin{aligned}
V \int_{-W}^{\infty} d F_{N}(x)+\sum_{l \in C} \int_{-W}^{-W+w_{l}}\left(x+W-w_{l}\right) d F_{N}(x) & \\
& +\sum_{l \in C} \int_{-\infty}^{+\infty} \ldots \int_{-\infty}^{+\infty} \delta\left(w_{-i}\right) d \mu\left(\left(w_{j}\right)_{j \notin C}\right),
\end{aligned}
$$

where $\mu$ is coalition $C$ 's common beliefs about the joint distribution of $\left(w_{j}\right)_{j \notin C}$ (from which $F_{N}($.$) is derived).$

The first order condition for maximization with respect to $w_{i}$ for $i \in C$ is

$$
\begin{aligned}
F_{N}(-W)+F_{N}(W)- & F_{N}\left(-W+w_{i}\right) \\
& +\sum_{l \in C, l \neq i} \int_{-\infty}^{+\infty} \ldots \int_{-\infty}^{+\infty}\left(\partial\left(\delta\left(w_{-l}\right)\right) / \partial w_{i}\right) d \mu\left(\left(w_{j}\right)_{j \notin c}\right)=0
\end{aligned}
$$

Equating (6.2) for two different agents $i$ and $i^{\prime}$ we obtain

$$
\begin{aligned}
& F_{N}\left(-W+w_{i}\right)-\sum_{l \in C, l \neq i} \int_{-\infty}^{+\infty} \cdots \int_{-\infty}^{+\infty}\left(\partial\left(\delta\left(w_{-l}\right)\right) / \partial w_{i}\right) d \mu\left(\left(w_{j}\right)_{j \notin c}\right) \\
& =F_{N}\left(-W+w_{i^{\prime}}\right)-\sum_{l \in C, l \neq i^{\prime}} \int_{-\infty}^{+\infty} \cdots \int_{-\infty}^{+\infty}\left(\partial\left(\delta\left(w_{-l}\right)\right) / \partial w_{i}\right) d \mu\left(\left(w_{j}\right)_{j \notin c}\right)
\end{aligned}
$$

Because the mechanism is impersonal, it is possible to subtract from both sides of (6.3) all the terms in the summations where $l=i$ and $l=i^{\prime}$. Afterwards the left-hand side of (6.3) is a function of $w_{i}$ and not of $w_{i}$, because the only term left in the summation is the one associated with $l=i^{\prime}$ and $w_{i}$, is not an argument of that function. Similarly the right-hand side of (6.3) is then a function of $w_{i}$, and not $w_{i}$.

Since the mechanism is impersonal, the two sides are the same functions of their respective arguments. Moreover, by the concavity of $\delta($.$) , they are both decreasing and$ can be equal only if the two arguments are equal.

It is easy to check that the second order conditions are fulfilled. \|

For simplicity, we impose the further restriction that $\delta$ is a function of the sum of everybody else's answer, i.e.

$$
\delta\left(x+\sum_{j \in C, j \neq i} w_{j}\right) .
$$

The following proof can be extended to the other symmetric cases but requires conditions on $\delta_{i}($.$) which are not easily interpreted.$

Definition 6. A differentiable Groves mechanism is said to be asymptotically pivotal if $\delta_{i}(y)$ approaches a constant $k$ as $y$ tends towards infinity and $\left|\delta_{i}^{\prime}(y)\right|$ is integrable.

Theorem 6.2. If $\sqrt{N} f_{N}(x)$ is uniformly bounded and converges at each point to a constant $K$, and $\sqrt{N} f_{N}^{\prime}(y)$ is continuous in y and converges to zero, then for an impersonal concave differentiable asymptotically pivotal Groves mechanism, the optimal global answer of a coalition of size $n$ approaches $n V$ (where $V=\sum_{i \in C} v_{i}$ ) as $N$ becomes large. 
Proof. The additional term in the analog of (4.7) is

$$
\begin{aligned}
(n-1) \int_{-\infty}^{+\infty} \delta^{\prime}\left(x+\frac{W(n-1)}{n}\right) & \sqrt{N} f_{N}(x) d x \\
& =(n-1) \int_{-\infty}^{+\infty} \delta^{\prime}(u) \sqrt{N} f_{N}\left(u-\frac{W(n-1)}{n}\right) d u
\end{aligned}
$$

since we can drop now the indices of the $\delta$.

Since $\sqrt{N} f_{N}(u-(W(n-1) / n))$ is uniformly bounded, $\left|\delta^{\prime}(u) \sqrt{N} f_{N}(u-(W(n-1) / n))\right|$ is bounded by an integrable function since $\left|\delta^{\prime}(u)\right|$ is integrable.

By Lebesgue's theorem:

$$
\lim _{N}(n-1) \int_{-\infty}^{+\infty} \delta^{\prime}(u) \sqrt{N} f_{N}(u-(W(n-1) / n)) d u=(n-1) K \int_{-\infty}^{+\infty} \delta^{\prime}(u) d u=0 .
$$

Consequently (6.4) can be made as small as wished by increasing $N$. Therefore, the result of Theorem 4.2 still holds. \|

The only mechanisms $\delta($.$) which are simultaneously concave and satisfy the assumption$ of asymptotically pivotal are the pivotal mechanisms plus a constant. However, the requirement of concavity is obviously a sufficient but not a necessary one to obtain the above results.

Finally, we can state, under the same assumptions as Theorem 6.2.

Theorem 6.3. With the normal distribution, $\forall \varepsilon>0, \forall \eta>0, \forall n<N^{\alpha}, \alpha<\frac{1}{6}, \exists N_{0}$ such that $N>N_{0}$ implies that the probability that there exists a coalition of size $n$ with a percapita gain larger than $\varepsilon$ is smaller than $\eta$.

Proof. The proof is similar to the proof of Section 5 but we have here an additional term in the gain attained by optimal cheating namely:

But

$$
S(n, N)=n \int_{-\infty}^{+\infty}[\delta(x+V(n-1))-\delta(x+(V(n-1) / n))] f_{N}(x) d x .
$$

$|\delta(x+V(n-1))-\delta(x+(V(n-1) / n))|<\left((n-1)^{2} / n\right) V \delta^{\prime}(x+\lambda V) \quad$ for $\quad \lambda \in[(n-1) /(n), n]$.

In per-capita terms

$$
\begin{aligned}
(S(n, N) / n) & <\int_{-\infty}^{+\infty}\left((n-1)^{2} / n\right) V\left|\delta^{\prime}(u)\right| f_{N}(u-\lambda V) d u \\
& <\int_{-\infty}^{+\infty} V_{\max }(n-1)^{2}\left|\delta^{\prime}(u)\right| f_{N}(u-\lambda V) d u
\end{aligned}
$$

As in Theorem 5.1 we have with arbitrary high probability

Taking

$$
V_{\max }<N^{\delta} \text { for any } \delta>0 \text {. }
$$

we obtain

$$
n<N^{\frac{1}{2}-\delta} \text { for } \delta>0
$$

$$
\begin{aligned}
(S(n, N) / n) & <\int_{-\infty}^{+\infty} N^{-\delta} \sqrt{N}\left|\delta^{\prime}(u)\right| f_{N}(u-\lambda V) d u \\
& <\alpha N^{-\delta}, \text { for some constant } \alpha,
\end{aligned}
$$

since $\left|\delta^{\prime}().\right|$ is integrable and $\sqrt{N} f_{N}($.$) converges uniformly to a constant. Hence the$ result, arguing as in Theorem 5.1. $\|$ 


\section{CONCLUSION}

The purpose of this section is to give an interpretation of the preceding results. We have shown that the probability that there exists a coalition of size $n$ less than the sixth root of the population size with a per-capita gain of more than $\varepsilon$, can be made as small as we wish with large $N$.

The requirement of a strictly positive per-capita gain can be justified by the need for communication to form coalitions. Communication costs are often thought to be of the order of the square of population size. Here we have taken the rather pessimistic point of view that they are only proportional to the size of the coalition; this neglects the costs of trial and error in the process of finding a viable coalition.

What about coalitions of size larger than the sixth root of the population size? First, these coalitions will clearly have free rider problems of their own. Moreover, they will have very high enforcement costs. We could have interpreted the required percapita gain, as a necessary enforcement cost for members of the coalition to be sure that the binding agreement they made is respected. For large populations, this type of cost becomes unbearable especially in a society which punishes recognized coalitions. Also, the large coalitions, because the optimal method of cheating is grossly to exaggerate, will be easily recognized. Of course, they could try to take this risk of discovery into account, but the attainable gain from cheating would be even smaller. Finally, it would be very easy for a society to catch such coalitions by infiltration.

Consequently for all these reasons very large coalitions should not be a problem. For small coalitions, our result indicates that manipulation by coalition is not likely to create difficulties for social decision making based on an appropriate Groves-type procedure.

The expected gain is:

\section{APPENDIX}

$$
\begin{aligned}
G=V \int_{-n V}^{-V} d F_{N}(x)+n \int_{-n V}^{-(n-1) V}[ & x+(n-1) V] d F_{N}(x) \\
& -n \int_{-V}^{-((n-1) V / n)}[x+((n-1) V / n) V] d F_{N}(x) .
\end{aligned}
$$

With the normal distribution we have:

$$
d F_{N}(x)=\frac{1}{\sqrt{2 \pi N}} e^{-\frac{1}{2} \frac{x^{2}}{N}} d x .
$$

The integral (1) can broken up in three parts A, B, C.

$$
\begin{aligned}
& \mathrm{A}=V \int_{-n V}^{-V} \frac{1}{\sqrt{2 \pi N}} \cdot e^{-\frac{1}{2} \frac{x^{2}}{N}} d x \leqq \frac{(n-1) V^{2}}{\sqrt{2 \pi N}} \cdot e^{-\frac{1}{2} \frac{V^{2}}{N}} \\
& \mathrm{~B}=n \int_{-n V}^{-(n-1) V}[x+(n-1) V] \frac{1}{\sqrt{2 \pi N}} e^{-\frac{x^{2}}{N}} d x \\
& \leqq n(n-1) V^{2} \frac{1}{\sqrt{2 \pi N}} e^{-\frac{\left(\frac{(n-1)^{2} V^{2}}{N}\right.}{2}}+\frac{\sqrt{N \cdot n}}{\sqrt{2 \pi}} \int_{-n V}^{-(n-1) V} \frac{x}{N} e^{-\frac{1}{2} \frac{x^{2}}{N}} d x \\
& \leqq n(n-1) V^{2} \frac{1}{\sqrt{2 \pi N}} e^{-\frac{1}{2} \frac{(n-1)^{2} V^{2}}{N}}+\frac{n \sqrt{N}}{\sqrt{2 \pi}}\left[e^{-\frac{n^{2} V^{2}}{2 N}}-e^{-\frac{(n-1)^{2} V^{2}}{2 N}}\right]
\end{aligned}
$$


Hence

$$
\begin{aligned}
\mathrm{C} & =-n \int_{-V}^{-((n-1) V / n)}[x+((n-1) V / n)] d F_{N}(x) \\
& \leqq-\frac{(n-1)}{n} V^{2} \frac{e^{-\frac{V^{2}}{2 N}}}{\sqrt{2 \pi N}}-\frac{n \sqrt{N}}{\sqrt{2 \pi}}\left[e^{-\frac{V^{2}}{2 N}}-e^{-\frac{(n-1)^{2}}{n^{2}} \frac{V^{2}}{2 N}}\right] .
\end{aligned}
$$

$$
\begin{aligned}
G \leqq \frac{(n-1) V^{2}}{\sqrt{2 \pi N}}+\frac{n(n-1) V^{2}}{\sqrt{2 \pi N}}-\frac{(n-1) V^{2}}{n \sqrt{2 \pi N}} & \\
& +\frac{n \sqrt{N}}{\sqrt{2 \pi}}\left[e^{-\frac{n^{2} V^{2}}{2 N}}-e^{-\frac{(n-1)^{2} V^{2}}{2 N}}-e^{-\frac{V^{2}}{2 N}}-e^{-\frac{(n-1)^{2} V^{2}}{2 n N}}\right]
\end{aligned}
$$

By using a Taylor expansion of the bracketed expression above, one obtains, under the conditions

$$
n \leqq N^{\frac{1}{6}-\delta}, V \leqq N^{\frac{1}{6}},
$$

that $G$ is bounded above by an expression where the dominant term is of the order of $n^{2} V^{2} / \sqrt{N}$.

First version received February 1977; final version accepted August 1977 (Eds.).

\section{REFERENCES}

BENNETT, E. and CONN, D. (1976), “ The Group Incentive Properties of Mechanisms for the Provisions of Public Goods" (mimeo).

CLARKE, E. H. (1971), "Multipart Pricing of Public Goods ", Public Choice, 2, 19-33.

DAVID, H. A. (1970), Order Statistics (Wiley).

GREEN, J, and LAFFONT, J, J. (1976a), "Révélation des Préférences pour les Biens Publics, Caractérisation des Mécanismes Satisfaisants ", D.P. Ecole Polytechnique n ${ }^{\circ}$ A 125 0176, à paraître dans les Cahiers du Séminaire d'Econométrie (Paris: C.N.R.S.).

GREEN, J. and LAFFONT, J. J. (1976b), "A Sampling Approach to the Free Rider Problem" (to appear in the Proceedings of the Kyriat Anavin Conference in Public Economics).

GREEN, J. and LAFFONT, J. J. (1977), “ Characterization of Satisfactory Mechanisms for the Revelation of Preferences for Public Goods ", Econometrica, 45, 427-438.

GROVES, T. (1973), "Incentives in Teams ", Econometrica, 41, 617-631.

HURWICZ, L. (1975), “ On the Existence of Allocation Systems whose Manipulative Nash Equilibria are Pareto Optimal "' (presented at the 3rd World Congress of the Econometric Society).

VICKREY, W. (1961), "Counterspeculation, Auctions and Competitive Sales Tenders", Journal of Finance, 16, 8-37.

WALKER, M. (1976), "On the Impossibility of a Dominant-Strategy Mechanism to Optimally Decide Public Goods Questions " (Department of Economics W.P. 160, S.U.N.Y. at Stony Brook). 
http://www.jstor.org

\section{LINKED CITATIONS}

- Page 1 of 1 -

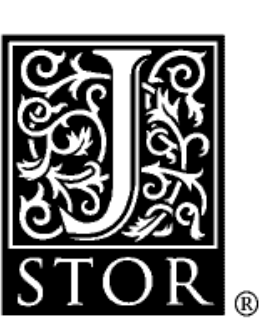

You have printed the following article:

\section{On Coalition Incentive Compatibility}

Jerry Green; Jean-Jacques Laffont

The Review of Economic Studies, Vol. 46, No. 2. (Apr., 1979), pp. 243-254.

Stable URL:

http://links.jstor.org/sici?sici=0034-6527\%28197904\%2946\%3A2\%3C243\%3AOCIC\%3E2.0.CO\%3B2-C

This article references the following linked citations. If you are trying to access articles from an off-campus location, you may be required to first logon via your library web site to access JSTOR. Please visit your library's website or contact a librarian to learn about options for remote access to JSTOR.

\section{References}

Characterization of Satisfactory Mechanisms for the Revelation of Preferences for Public

\section{Goods}

Jerry Green; Jean-Jacques Laffont

Econometrica, Vol. 45, No. 2. (Mar., 1977), pp. 427-438.

Stable URL:

http://links.jstor.org/sici?sici=0012-9682\%28197703\%2945\%3A2\%3C427\%3ACOSMFT\%3E2.0.CO\%3B2-Q

\section{Incentives in Teams}

Theodore Groves

Econometrica, Vol. 41, No. 4. (Jul., 1973), pp. 617-631.

Stable URL:

http://links.jstor.org/sici?sici=0012-9682\%28197307\%2941\%3A4\%3C617\%3AIIT\%3E2.0.CO\%3B2-E

\section{Counterspeculation, Auctions, and Competitive Sealed Tenders}

William Vickrey

The Journal of Finance, Vol. 16, No. 1. (Mar., 1961), pp. 8-37.

Stable URL:

http://links.jstor.org/sici?sici=0022-1082\%28196103\%2916\%3A1\%3C8\%3ACAACST\%3E2.0.CO\%3B2-L 